\title{
Metabolic disorders and gut microbiota ${ }^{\star}$
}

\author{
A. D. Kotrova, A. N. Shishkin, M. V. Lukashenko \\ St. Petersburg State University, \\ 7-9, Universitetskaya nab., St. Petersburg, 199034 Russian Federation
}

For citation: Kotrova A. D., Shishkin A. N., Lukashenko M. V. Metabolic disorders and gut microbiota. Vestnik of Saint Petersburg University. Medicine, 2020, vol. 15, issue 3, pp. 177-183.

https://doi.org/10.21638/spbu11.2020.302

Obesity, type 2 diabetes mellitus, metabolic syndrome are metabolic widespread disorders that arise both under the influence of external factors (physical inactivity, high-calorie diet) and under the influence of internal factors. The latter includes the intestinal microbiota which deserves more and more attention in developing new strategies for the correction of metabolic diseases. The discovery of new approaches for the gut microbiota study (metagenomic, metabolomic) gives a new insight into the diversity and involvement of intestinal bacteria in the metabolic processes of the whole organism. This article are reviewed the mechanisms of the gut bacteria impact on lipid and carbohydrate metabolism, the relationship of bacteria species and their metabolites with tissue insulin sensitivity, body mass index. Special attention in the regulation of tissue insulin sensitivity is paid to the role of short-chain fatty acids and secondary bile acids, which are metabolites of gut bacteria. Understanding the influence of human microbiota and its metabolites on lipid and carbohydrate metabolism provides the basis for the development of new approaches to the prevention and treatment of socially significant metabolic diseases such as type 2 diabetes mellitus, obesity, metabolic syndrome.

Keywords: microbiota, metabolic disorders, lipopolysaccharide, short-chain fatty acids, bile acids.

\section{Introduction}

Metabolic diseases have been a global problem for the last century all over the world. Thus, the prevalence of obesity in some developed countries reaches pandemic levels, and for type 2 diabetes mellitus (DM), there is a progressive incidence increase annually.

Metabolic syndrome (MS) is presented in a quarter of the adult population in the world and has become a serious public health problem every year, especially in urban areas. In addition to genetic predisposition, there are such important external factors as low physical activity and high-calorie diet, which contribute to the development of metabolic disorders [1].

In recent years, many omics technologies studies have been conducted, the results of which indicate a possible link between qualitative and quantitative changes in the gut microbiota composition and the development of obesity, DM, MS [2].

The gut microbiota, with its vast and unique genome, profoundly influence on human metabolism. First, it helps in metabolic pathways functioning by the synthesis of essential vitamins (especially group B); secondly, gut bacteria, through the fermentation of indi-

* The reported study was funded by RFBR, project number 20-315-90106.

(C) St. Petersburg State University, 2020 
gestible carbohydrates, provide the human organism with short-chain fatty acids (SCFA), which are either a source of energy, or act as signaling molecules by binding to membrane receptors for the integration of metabolic functions [3].

European-style diets rich in saturated fats, simple carbohydrates and devoid of dietary fiber is considered as altering the structure of the gut microbiota factor, which adversely affects gut permeability and colonization by metabolic disorders-causing gut bacteria.

In this article are reviewed the mechanisms of the gut bacteria influence on the human metabolism, the links of bacteria and their metabolites with body mass index, insulin sensitivity and lipid metabolism. Understanding these processes and correlations provides the basis for the development of new approaches to the prevention and treatment of socially significant metabolic diseases such as DM, obesity, MS.

\section{Microbiota and its composition}

Microbiota is a term used to characterize the microbiocenosis of organ systems. So, the gastrointestinal tract is the largest habitat of bacteria and includes more than 50 genera and more than 500 species of microorganisms, the number of which gradually increases along the intestine, reaching a maximum value in the colon, which is approximately $10^{9}-$ $10^{11} \mathrm{CFU} / \mathrm{ml}$ [4-7]. This gradient is mainly due to the rapid luminal flow, low $\mathrm{pH}$ in the upper gastrointestinal tract, and the secretion of antibacterial substances such as bile acids [8].

The main gut microbiota bacterial types are Firmicutes, Bacteroidetes, Actinobacteria, Proteobacteria, Verrucomicrobia, Fusobacteria, and Euryarchaeota (Table) [2; 9]. Phylums Firmicutes and Bacteroidetes characterized by the highest species diversity and account for $90 \%$ of the total gut bacteria number [10]. The species diversity of bacteria varies in different parts of the intestine $[11 ; 12]$. Thus, in the small intestine is dominated Firmicutes (Lactobacillaceae) and Proteobacteria (Enterobacteriaceae), and in the colon - Bacteroidetes (Bacteroidaceae, Prevotellaceae, Rikenellaceae), Firmicutes (Lachnospiraceae, Ruminococcaceae) and only representative Verrucomicrobia - Akkermansia muciniphila [13].

Table. Bacteria in the human gut microbiota $[2 ; 10 ; 15]$

\begin{tabular}{|l|l|c|}
\hline \multicolumn{1}{|c|}{ Phylum } & Gram-stain & Genus or species \\
\hline Bacteroidetes & Gram (-) & $\begin{array}{r}\text { Bacteroides, Parabacteroides, Prevotella, Porphyromonas, Odoribacter, } \\
\text { Barnesiella, Alistipes }\end{array}$ \\
\hline Firmicutes & Gram (+) & $\begin{array}{r}\text { Lactobacillus, Ruminococcus, Mycoplasma, Enterococcus, } \\
\text { Streptococcus, Staphylococcus, Clostridium cocleatum, Roseburia, } \\
\text { Oscillibacter spp., Intestinibacter spp., Faecalibacterium prausnitzii, } \\
\text { Blautia, Dorea, Coprococcus }\end{array}$ \\
\hline Proteobacteria & Gram (-) & Escherichia coli, Escherichia spp., Proteus, Pseudomonas \\
\hline Actinobacteria & Gram (+) & Bifdobacteria spp. \\
\hline Verrucomicrobia & Gram (-) & Akkermansia muciniphila \\
\hline
\end{tabular}

The functions of the intestinal microbiota are numerous and varied: these are protective (colonization resistance), regulation of the immune, endocrine and nervous systems, 
metabolism of nutrients and xenobiotics, synthesis of vitamins (folates, vitamin $\mathrm{K}$, biotin, riboflavin (B2), cobalamin (B12)), bile acids, biologically active compounds and detoxication [11]. These processes play an essential role in the metabolism of the human host. The development of new methods for studying the intestinal microbiota (RT-PCR, omics technologies) gave the new insight into the diversity and impact of gut bacteria to the metabolic processes of the whole organism. In this regard, in the last decade, the gut microbiota has received more and more attention as a new factor involved in the pathophysiology of metabolic diseases $[14 ; 15]$.

\section{Mechanisms of gut microbiota influence on metabolic processes in the human body}

The most important factor determining the composition and metabolism of the gut microbiota in the modern world is diet and food additives [16]. In a 2015 American study, it was shown that emulsifiers (carboxymethylcellulose, polysorbate-80), widely used in food, caused nonspecific inflammation, obesity and MS in normal mice. These compounds altered the gut microbial composition and increased its pro-inflammatory potential. Transplantation of fecal microbiota processed with an emulsifier to gnotobiont mice led to the phenotype of obese mice, which confirms the impact of these additives to the development of metabolic disorders [17].

Consumption of a high-fat diet leads to disruption of the gut microbiota composition by inducing the death of gram-negative bacteria (mainly Bacteroidetes and Proteobacteria), thus promoting the release of large amounts of lipopolysaccharide (LPS), which is an integral part of their cell wall [18]. In case of the intestinal barrier violation, the permeability of the mucous membrane increases, and LPS from the large intestine is transported in greater quantities into the bloodstream through the intercellular spaces in the intestinal wall. LPS mediates the formation of the CD-14 complex with Toll-like receptor- 4 of macrophages and endothelial cells, which causes the release of pro-inflammatory cytokines: interleukin-1 (IL-1), interleukin-6 (IL-6), TNF- $\alpha$ [2; 19; 20]. The above changes lead to the development of systemic low-grade inflammation and, as a consequence, a decrease in insulin sensitivity, increased lipogenesis in the liver, and activation of inflammation in adipose tissue [19].

In addition to the influence of external factors, several mechanisms of gut microbiota influence on the metabolism of the human host have been studied. When polysaccharides are broken down with the participation of microbiota to easily assimilated monosaccharides, either they are absorbed by enterocytes, or they are fermented by the microbiota with the formation of short-chain fatty acids (SCFA) - acetic, propionic, butyric acids. Subsequently, SCFAs are delivered through the portal vein system to the liver, where they serve as substrates for gluconeogenesis and de novo lipogenesis. According to A. A. Kurmangulov study, obese people have the decrease number of SCFAs, and the absolute level of all SCFAs had an inverse moderate correlation with the BMI value. Considering that the main producers of SCFA in the gut are obligate firmicutes anaerobes, it can be assumed that with an increase in BMI, there is a decrease in the metabolic activity of lactic acid microorganisms, mainly bifidobacteria and lactobacilli. Thus, the lowest values of the SCFA are determined in the group with 3rd degree obesity [21].

SCFAs are ligands for G-protein-coupled receptors (GPCRs) such as GPR41, GPR43 and GPR109A, which are expressed in intestinal neuroendocrine cells. These spe- 
cialized cells perform important endocrine functions in the intestines and pancreas. In the production of SCFA, GPCR stimulate the tyrosine-tyrosine peptide (YY-peptide), which slows down intestinal motility and thus facilitates the absorption of nutrients $[19 ; 22 ; 23]$. Thus, in patients receiving propionic acid therapy for 24 weeks, an increase in the production of glucagon-like peptide 1 (GLP-1) and YY-peptide was observed, which reduced appetite, increased tissue insulin sensitivity, and reduced fat deposition [23].

In a study by Perry R. J., Peng L. et al., increased acetate production by altered gut microbiota in high-fat rodents leads to increased ghrelin secretion and glucose-stimulated insulin secretion. As a result, a positive feedback loop is created, which leads to hyperphagia, hypertriglyceridemia, ectopic lipid deposition in the liver and skeletal muscles, and insulin resistance in the liver and muscles [24].

Altered gut microbiota is also capable of suppressing the fasting-induced adipose factor expression (FIAF, or angiopoietin-like protein -4) in enterocytes. FIAF is a potent inhibitor of lipoprotein lipase in adipose tissue. As a result, the breakdown of triacylglycerol-containing lipoproteins into free fatty acids and the subsequent deposition of triglycerides in adipose tissue is activated. In addition, gut microbiota inhibits the release of adenosine monophosphate, an activated protein kinase (AMPK), expressed mainly in skeletal muscle, brain and liver in response to metabolic stress (hypoxia, physical activity). This leads, on the one hand, to inhibition of mitochondrial fatty acid oxidation, ketogenesis, glucose uptake and insulin secretion, and, on the other hand, to an increase in lipogenesis, cholesterol and triglyceride synthesis [20;25].

The intestinal microbiota is also involved in the metabolism of bile acids (FA), the violation of which is associated with changes in lipid metabolism and the development of metabolic diseases. With dysbiosis, the formation of secondary FAs and the beneficial effects caused by them decrease. Secondary FAs activate the secretion of GLP-1 through membrane FA receptors (TGR-5 receptors), which leads to an increase in glucose tolerance, an increase in energy consumption by brown adipose tissue and skeletal muscle [2]. In addition, secondary FAs formed as a result of dehydroxylation of primary FAs by normal microbiota (for example, Clostridium scindens) have antimicrobial effect, forming colonization resistance, which prevents colonization of the intestine by pathogenic microorganisms [26].

The above data undoubtedly indicates that gut microbiota can play an important role in the prevention or development of metabolic disorders. But for this, it is necessary to confirm that patients with metabolic diseases have dysbiosis, i. e. qualitative and quantitative changes of microbiota composition, which determine a wide range of metabolic disorders, and which would show that there are changes in the gut microbiota ecology that cause the development of obesity and associated disorders, and not vice versa [20].

Early studies in this area indicated an increased Firmicutes/Bacteroidetes ratio in obese individuals. However, in subsequent studies using shotgun sequencing, no significant difference was found $[2 ; 3 ; 25]$. In other studies, an increase in the number of Actinobacteria associated with obesity with a decrease in the number of Bacteroidetes and a constant number of Firmicutes was obtained [27]. Studies using metagenomics methods have found that obesity is associated with a decrease in the number of bacterial genes or the bacteria diversity [3]. Thus, there is currently no consensus on changes in the composition of gut microbiota in obesity [5]. 
According Santos - Marcos J.A. in patients with MS, bacteria with saccharolytic activity Bacteroides fragilis group, Parabacteroides distasonis, Bacteroides thetaiotaomicron and Ruminococcus flavefaciens subgroup are reduced. This leads to a decrease in the production of SCFA (propionate and acetate), which entails a decrease in beneficial bacteria such as Faecalibacterium prausnitzii , Eubacterium rectale, and Fusobacterium nucleatum [28].

According to Liu R. et al., In obese individuals, the number of Bacteroides thetaiotaomicron, which ferments glutamate, is significantly reduced, and its concentration in serum is increased [29]. There is also a decrease in the number of Oscillospira, Christensenellacea, Rikenellaceae, the genus Bifidobacterium and mucin-degrading bacteria Akkermansia muciniphila (type Verrucomicrobia). Akkermansia muciniphila has an inversely proportional link with the body mass index and the level of triglycerides in the blood plasma [3; 7].

In in vitro and in vivo studies, butyrate-producing bacteria such as Eubacterium, Fusobacterium, Anaerostipes, Roseburia, Subdoligranulum and Faecalibacterium have been observed to have anti-inflammatory potential to reduce bacterial translocation, improve tight junction function, and stimulate mucin secretion for intestinal wall [30].

It was also recently confirmed that some species of the genus Lactobacillus may be associated with obesity [28]. Thus, Lactobacillus ingluviei and Lactobacillus acidophilus are associated with weight gain, while Lactobacillus casei/paracasei, Lactobacillus plantarum and Lactobacillus gasseri with its decline [2].

In patients with DM was observed low levels of bifidobacteria and butyrate-producing bacteria, in particular Faecalibacterium prausnitzii, genus Roseburia negatively correlated with BMI, fasting hyperglycemia, cholesterol, TG $[28 ; 31 ; 32]$.

One large Mexican study (1065 people) found an increase in Prevotella copri, Ruminococcus callidus, Prevotella stercorea and a decrease in Bacteroides in MS patients. At the same time, in gut microbiota of people with normal weight prevailed Bacteroides uniformis, Vellionella parvula, Bacteroides fragilis, Bacteroides ovatus, Bacteroides acidifaciens, Alistipes massilensis, Roseburia faecis, E. coli [1].

In persons with MS, an increase of total bacterial mass was revealed due to the growth of opportunistic bacteria, primarily Eubacteriumlentum, Clostridium hystolyticum and propionicum, Nocardia, Bacilluscereus, Prevotella, fam. Enterobacteriaceae against the decrease in the amount of beneficial ones [33].

\section{Conclusion}

The intestinal microbiota, its genetic material and metabolites are an important part of the human metabolism. The transformation of organic compounds supplied with food, the degree of their assimilation and the effect on human organs and tissues partly depend on the qualitative and quantitative composition of the gut microbiota. Studies on animals have shown the mechanisms of the gut microbiota effect on BMI, lipid and carbohydrate metabolism. In large people studies, certain correlations of types, genera, species of bacteria with signs of MS have been shown, however, the data are rather diverse and contradictory. Further study of the bacteria genome and metabolome, link confirmation of already known bacteria with the development of obesity, DM, MS will allow developing new approaches to the prevention and treatment of metabolic diseases. 


\section{References}

1. Guevara-Cruz M., Flores-López A. G., Aguilar-López M., Sánchez-Tapia M., Medina-Vera I., Díaz D., Tovar A. R., Torres N. Improvement of Lipoprotein Profile and Metabolic Endotoxemia by a Lifestyle Intervention That Modifies the Gut Microbiota in Subjects with Metabolic Syndrome. J. Am. Heart Assoc., 2019, vol. 8, no. 17, article ID 012401.

2. Pokrovskaya E. V., Shamkhalova M.Sh., Shestakova M. V. New views on the state of the intestinal microbiota in obesity and type 2 diabetes. Sakharnyi diabet, 2019, vol. 22, no. 3, pp. 253-262. (In Russian)

3. Iacobini C., Pugliese G., Blasetti Fantauzzi C., Federici M., Menini S. Metabolically healthy versus metabolically unhealthy obesity. Metabolism, 2019, vol. 92, pp. 51-60.

4. Borshchev Y.Y., Ermolenko E. I. Metabolic syndrome and intestinal microecology. Transliatsionnaia meditsina, 2014, no. 1, pp. 19-28. (In Russian)

5. Kravchuk E. N., Neymark A.E., Grineva E.N., Galagudza M.M. The role of gut microbiota in metabolic regulation. Sakharnyi diabet, 2016, vol. 19, no. 4, pp. 280-285. (In Russian)

6. Plotnikova E. Y., Krasnov O. A. Metabolic syndrome and intestinal micro flora: what overall? Eksperimentalnaia i klinicheskaia gastroenterologia, 2015, vol. 112, no. 12, pp. 64-73. (In Russian)

7. Eckburg P. B., Bik E. M., Bernstein C. N., Purdom E., Dethlefsen L., Sargent M., Gill S. R., Nelson K. E., Relman D. A. Diversity of the human intestinal microbial flora. Science, 2005, vol. 308, no. 5728, pp. 1635-1638.

8. O'Hara A.M, Shanahan F. The gut flora as a forgotten organ. EMBO Reports, 2006, vol. 7, no. 7, pp. 688-693.

9. Rajilić-Stojanović M., de Vos W.M. The first 1000 cultured species of the human gastrointestinal microbiota. FEMS Microbiol. Rev., 2014, vol. 38, no. 5, pp. 996-1047.

10. Tap J., Mondot S., Levenez F., Pelletier E., Caron C., Furet J.P., Ugarte E., Muñoz-Tamayo R., Paslier D. L., Nalin R., Dore J., Leclerc M. Towards the human intestinal microbiota phylogenetic core. Environ. Microbiol., 2009, vol. 11, no. 10, pp. 2574-2584.

11. Jandhyala S. M., Talukdar R., Subramanyam C., Vuyyuru H., Sasikala M., Nageshwar Reddy D. Role of the normal gut microbiota. World J. Gastroenterol., 2015, vol. 21, no. 29, pp. 8787-8803.

12. Sekirov I., Russell S. L., Antunes L. C., Finlay B. B. Gut microbiota in health and disease. Physiol. Rev., 2010, vol. 90, no. 3, pp. 859-904.

13. Belzer C., de Vos W. M. Microbes inside - from diversity to function: the case of Akkermansia. ISME J., 2012, vol. 6, no. 8, pp. 1449-1458.

14. Tremaroli V., Backhed F. Functional interactions between the gut microbiota and host metabolism. Nature, 2012, vol. 489, no. 7415, pp. 242-249.

15. Erejuwa O. O., Sulaiman S.A., Ab Wahab M.S. Modulation of gut microbiota in the management of metabolic disorders: the prospects and challenges. Int. J. Mol. Sci., 2014, vol. 15, no. 3, pp. 4158-4188.

16. Zhernakova A., Kurilshikov A., Bonder M.J., Tigchelaar E. F., Schirmer M., Vatanen T., Mujagic Z., Vich Vila A., Falony G., Vieira-Silva S., Jun Wang, Imhann F., Brandsma E., Jankipersadsing S. A., Joossens M., Cenit M. C., Deelen P., Swertz M. A., Weersma R. K., Feskens E. J. M., Netea M. G., Gevers D., Jonkers D., Franke L., Aulchenko Yu. S., Huttenhower C., Raes J., Hofker M. H., Xavier R. J., Wijmenga C., Jingyuan Fu. Population-based metagenomics analysis reveals markers for gut microbiome composition and diversity. Science, 2016, vol. 352, no. 6285, pp. 565-569.

17. Chassaing B., Koren O., Goodrich J.K., Poole A.C., Srinivasan S., Ley R.E., Gewirtz A.T. Dietary emulsifiers impact the mouse gut microbiota promoting colitis and metabolic syndrome. Nature, 2015, vol. 519, no. 7541, pp. 92-96.

18. Neal M.D., Leaphart C., Levy R., Prince J., Billiar T.R, Watkins S., Jun Li, Cetin S., Ford H., Schreiber A., Hackam D. J. Enterocyte TLR4 mediates phagocytosis and translocation of bacteria across the intestinal barrier. J. Immunol., 2006, no. 176, pp. 3070-3079.

19. Aitbaev K. A., Murkamilov I.T. Obesity and metabolic syndrome: pathophysiological role of gut microbiota and potential of the alternative therapy. Complex Issues of Cardiovascular Diseases, 2017, no. 3, pp. 120-130.

20. Wang J., Li W., Wang C., Wang L., He T., Hu H., Song J., Cui C., Qiao J., Qing L., Li L., Zang N., Wang K., Wu C., Qi L., Ma A., Zheng H., Hou X., Liu F., Chen L. Enterotype Bacteroides is Associated with a High Risk in Patients with Diabetes: A Pilot Study. J. Diabetes Res., 2020, vol. 2020, article ID 6047145.

21. Kurmangulov A.A, Dorodneva E.F, Isakova D.N. Functional activity of intestinal microbiota with metabolic syndrome. Ozhirenie i metabolizm, 2016, vol. 13, no. 1, pp. 16-19. (In Russian) 
22. Faith J. J., Guruge J.L., Charbonneau M., Subramanian S., Seedorf H., Goodman A. L., Clemente J.C., Knight R., Heath A.C., Leibel R. L., Rosenbaum M., Gordon J. I. The long-term stability of the human gut microbiota. Science, 2013, vol. 341, no. 6141, pp. 1237439.

23. Chambers E.S, Viardot A., Psichas A., Morrison D. J., Murphy K.G., Zac-Varghese S.E. K., MacDougall K., Preston T., Tedford C., Finlayson G.S., Blundell J.E., Bell J.D., Thomas E. L., Mt-Isa S., Ashby D., Gibson G. R., Kolida S., Dhillo W.S., Bloom S. R., Morley W., Clegg S., Frost G. Effects of targeted delivery of propionate to the human colon on appetite regulation, body weight maintenance and adiposity in overweight adults. Gut., 2015, vol. 64, no. 11, pp. 1744-1754.

24. Perry R. J., Peng L., Barry N. A, Cline G. W., Zhang D., Cardone R. L., Petersen K. F., Kibbey R. G., Goodman A.L., Shulman G. I. Acetate mediate a microbiome-brain- $\beta$-cell axis to promote metabolic syndrome. Nature, 2016, vol. 534, no. 7606, pp. 213-217.

25. Bäckhed F., Ding H., Wang T., Hooper L. V., Koh G. Y., Nagy A., Semenkovich C. F., Gordon J. I. The gut microbiota as an environmental factor that regulates fat storage. Proc. Natl. Acad. Sci. USA, 2004, vol. 101, no. 44, pp. 15718-15723.

26. Chaplin A.V., Rebrikov D. V., Boldyreva M.N. The human microbiome. Bulletin of Pirogov Medical University, 2017, no. 2, pp. 5-13.

27. Volovnikova V. A., Kotrova A. D., Ivanova K. A., Ermolenko E. I., Shishkin A. N. The role of gut microbiota in the development of obesity. Juvenis Scientia, 2019, no. 6, pp. 4-10. (In Russian)

28. Santos-Marcos J.A., Perez-Jimenez F., Camargo A. The role of diet and intestinal microbiota in the development of metabolic syndrome. Journal of Nutritional Biochemistry, 2019, no. 70, pp. 1-27.

29. Liu R., Hong J., Xu X., Feng Q., Zhang D., Gu Ya., Shi J., Zhao S., Liu W., Wang X., Xia H., Liu Z., Cui B., Liang P., Xi L., Jin J., Ying X., Wang X., Zhao X., Li W., Jia H., Lan Z., Li F., Wang R., Sun Y., Yang M., Shen Y., Jie Z., Li J., Chen X., Zhong H., Xie H., Zhang Y., Gu W., Deng X., Shen B., Xu X., Yang H., Xu G., Bi Y., Lai S., Wang J., Qi L., Madsen L., Wang J., Ning G., Kristiansen K., Wang W. Affiliations expand Gut microbiome and serum metabolome alterations in obesity and after weight-loss intervention. Nat. Med., 2017, vol. 23, no. 7, pp. 859-868.

30. Ganesan K., Chung S. K., Vanamala J., Xu B. Causal Relationship between Diet-Induced Gut Microbiota Changes and Diabetes: A Novel Strategy to Transplant Faecalibacterium prausnitzii in Preventing Diabetes. Int. J. Mol. Sci., 2018, vol. 19, no. 12, article ID 3720.

31. Gontsaryuk D., Khristich T. The role of microbiota in the course and development of metabolic processes in chronic pancreatitis. Bulletin of the Club of Pancreatologists, 2017, vol. 38, no. 4, pp. 22-26.

32. Kotrova A. D., Shishkin A. N., Semenova O. I., Slepykh L. A. The role of intestinal microbiota in the development of metabolic syndrome. Eksperimentalnaia i klinicheskaia gastroenterologia, 2019, vol. 172, no. 12, pp. 101-108.

33. Turdiev S.M. Metabolic syndrome - an urgent problem of the XXI century. Sbornik nauchnykh trudov po materialam Mezhdunarodnoi nauchnoi konferentsii, posviashchennoi 85-letiiu Kurskogo gosudarstvennogo meditsinskogo universiteta. Kursk, Kurskii gosudarstvennyi meditsinskii universitet Publ., 2020, pp. 304-308.

Received: October 20, 2020 Accepted: November 30, 2020

Authors'information:

Anna D. Kotrova — Junior Researcher, Postgraduate Student; anna_hoh@mail.ru Alexandr N.Shishkin - Professor, MD; alexshishkin@bk.ru MariaV.Lukashenko _ Student; pushisti.legolas@mail.ru 